\title{
Compute Similarity of CAD Models Based on Bipartite Graph
}

\author{
Xue-Yao Gao ${ }^{1}$, Chun-Xiang Zhang ${ }^{2}$ and Zhi-Mao $\mathrm{Lu}^{3}$ \\ ${ }^{1}$ School of Computer Science and Technology, Harbin University of Science and \\ Technology, Harbin 150080, China \\ ${ }^{2}$ School of Software, Harbin University of Science and Technology, Harbin \\ 150080, China \\ ${ }^{3}$ School of Computer Science and Technology, Dalian University of Technology, \\ Dalian 116024, China \\ z6c6x666@163.com
}

\begin{abstract}
Model retrieval is widely applied to many fields including computer aided design, feature modeling and computer aided manufacturing. In order to retrieve a satisfied CAD model from a large model library, a new method to compute the similarity between two CAD models is presented in which a bipartite graph is used to match source faces with target faces. The number of edges in faces is extracted from CAD models. Based on the number of edges in faces, the similarity between source face and target face is computed. The maximum matching weight is calculated by KM algorithm. Then, the similarity between two CAD models is gotten. Several CAD models are given and their similarities are computed. Experimental results show that the method can evaluate the similarity between source CAD model and target CAD model efficiently.
\end{abstract}

Keywords: model retrieval, CAD model, bipartite graph, source faces, target faces

\section{Introduction}

The similarity of CAD models is the approximate degree between their component parts and their architectures. The similar models need be found from the existing model libraries in practical applications. The CAD model matching and the CAD model reusing are very important. Currently, the methods to compute similarities of CAD models can be divided into two categories. They are respectively the computation of local shape similarity and the computation of overall shape similarity.

Huang presents a retrieval method of 3D CAD models with the integration of manufacturing semantics. A 3D CAD model is transformed into semantic objects based on manufacturing features and the coupled map of manufacturing features is used to describe it. The complete bipartite graph is built whose nodes denote manufacturing features, and the similarity between these two 3D CAD models is calculated by the optimal matching algorithm [1]. Zhang proposes a retrieval approach of 3D CAD models based on ant colony algorithm, in which B-rep information of the CAD model is extracted and an attribute adjacent graph is used to represent this model. Ant colony algorithm is applied to detect common sub-graphs in attribute adjacent graph. The similarity of two CAD models is evaluated by comparing local similar features [2]. Tao gives a retrieval method of 3D CAD models in which Lagrange multiplier is used to solve the attribute adjacent graph [3]. Tao puts a retrieval method of CAD models based on non-exact match of attribute adjacent graphs. The method builds the similarity measure of two models and takes the measure as an optimization objective function which is applied to select the matching matrix of different vertexes [4]. Sun gives a retrieval method based on the topology approach. The spanning tree algorithm based on breadth-first search is used to 
obtain an initial boundary matching scheme between retrieval objects and retrieval conditions. Based on the optimal boundary matching scheme, the absolute error between retrieval objects and retrieval conditions is calculated [5]. Ma presents a retrieval method based on face shape codes. Based on shape codes of all faces in a model, a matrix is constructed which describes its overall shape. The matrix can effectively evaluate the similarity between two models under different accuracy requirements [6]. Bai proposes a local retrieval of 3D CAD models in which there are 3 partial retrieval modes. They are respectively normal retrieval; exact retrieval and relaxed retrieval which are defined to meet different local retrieval requirements for design reuse [7]. Liu gives a novel method to represent and retrieve 3D CAD models based on several voxelization algorithms. It is a content-based approach, which only depends on shapes and does not need any semantics or coding information [8]. Bosche presents a new approach to recognize and retrieve automatically 3D CAD objects in architecture, project and facility management [9]. Marefat proposes a method for retrieving CAD solid models in which the similarity-based matching and reusing plans are used. According to spatial relationships between functions, a signature is formed. With the signature, an indexing scheme is developed to store and retrieve digital components [10]. Li uses optimal match weights of 2D images to retrieve 3D CAD models. Phog descriptors are applied to describe 2D images projected from a model. Lagrange multipliers, vector quantization and support vector machine are used to assign adaptively an optimal weight to each projected image [11]. Hu gives a parallel global optimal approach of feedback for 3D CAD model retrieval under a novel unified mathematical model in which particle swarm optimization algorithm is utilized [12].

This paper presents a new method based on bipartite graph to compute the similarity between two models. The number of edges in faces is obtained, which is applied to calculate the similarity between source face and target face. Then, the bipartite graph matching method is used to compute the similarity between two models. Experimental results show that the method can calculate effectively the similarity between two models.

\section{The Similarity of Faces}

The attribute adjacent graph can reflect topological information and geometrical information in models. The information about faces, edges and vertexes can be obtained from models. A CAD model is represented by attribute adjacent graph which can accurately reflect characteristics of this model such as parallel relationship and vertical relationship between two faces. A CAD model is shown in Figure 1. Figure 2 is its attribute adjacent graph.

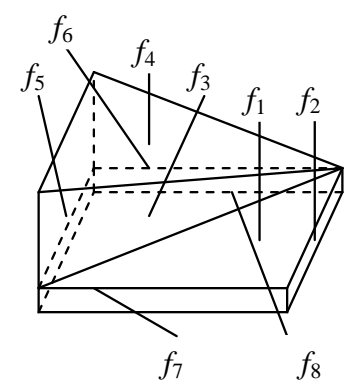

Figure 1. CAD Model

The attribute adjacent graph is denoted as $\mathrm{G}=(\mathrm{V}, \mathrm{E})$. V is the set of vertexes in graph. $\mathrm{E}$ is the set of nodes in graph. Face $f_{\mathrm{i}}$ in CAD model corresponds to a unique node $v_{\mathrm{i}}$ in G. If face $f_{\mathrm{i}}$ is adjacent with face $f_{\mathrm{j}}$ in CAD model, there is a corresponding edge between $v_{\mathrm{i}}$ and $v_{\mathrm{j}}$ in $\mathrm{G}$. 


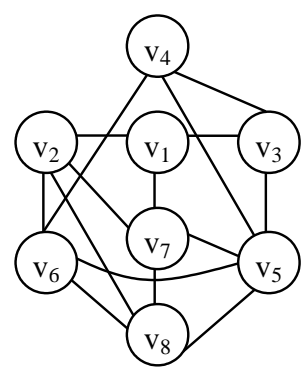

Figure 2. Attribute Adjacent Graph

The attribute adjacent graph describes locations of vertexes, edges and faces. At the same time, their relationships are also described. A CAD model is composed of faces. A face is composed by some edges. An edge is composed of vertices. To compute the similarity between two CAD models can be converted to calculate similarities between their corresponding faces. The similarity between two faces is the sum of similarities between their corresponding edges. So, the number of faces and the number of edges are used to calculate the similarity between two models. Different plane graphs have different number of edges. A plane circle has 2 edges. A plane triangle has 3 edges. A plane quadrilateral has 4 edges. There are various types of CAD models and a model has different shape faces. The number of vertexes of faces is large. The number of edges in faces is selected as features to compute the similarity of two models. Without considering other features, the method reduces the size of the similarity computation. The similarity of cylindrical faces, conical faces, spherical faces, convexes and concaves can be calculated based on their number of edges.

The number of edges has an influence on face shape. A face is composed of edges. Therefore, the number of edges is used to measure shape differences between two faces. The similarity between face $f_{i}$ and face $f_{j}$ is calculated by equation (1). $\operatorname{Num}(X)$ is the number of edges in face $X$, here.

$$
\operatorname{Sim}_{\text {face }}\left(f_{i}, f_{j}\right)=1-\frac{\left|\operatorname{Num}\left(f_{i}\right)-\operatorname{Num}\left(f_{j}\right)\right|}{\max \left(\operatorname{Num}\left(f_{i}\right), \operatorname{Num}\left(f_{j}\right)\right)}
$$

Here, target face is a plane quadrilateral. At the same time, source faces are respectively plane circle, plane triangle, plane quadrilateral, plane hexagon and plane octagon. Equation (1) can be used to compute the similarity between source face and target face. The results are shown in Table 1.

Table 1. Similarity between Source Face and Target Face

\begin{tabular}{ccc}
\hline Graphs & The number of edges & Similarity \\
\hline Plane circle & 2 & 0.500 \\
Plane triangle & 3 & 0.750 \\
Plane quadrilateral & 4 & 1.000 \\
Plane hexagon & 6 & 0.333 \\
Plane octagon & 8 & 0.500 \\
\hline
\end{tabular}

In CAD models, a quadrilateral is a common graph. From Table 1, it can be seen that a plane circle has 2 edges and its similarity is 0.500 . The number of edges in a plane triangle is 3 and its similarity is 0.750 . A triangle is similar with a quadrilateral in shape. A plane quadrilateral has 4 edges and its similarity is 1.000. A quadrilateral is very similar with a quadrilateral in shape. The number of edges in a plane hexagon is 6 and its similarity is 0.333 . A plane octagon has 8 edges and its similarity is 0.500 . They are not similar with a quadrilateral in shape. According to similarities, it can be seen that a quadrilateral is closest to a quadrilateral in shape. A triangle is closer to a quadrilateral in shape. A circle and an octagon are close to a quadrilateral in shape. 
For regular faces, the number of edges is easily calculated and the information of edges is easily extracted from faces. For irregular faces, there are some arcs. For example, there are arcs in conical face. The arc can be regarded as an edge.

\section{Calculate the Similarity of Models based on Bipartite graph}

In this paper, the number of edges in faces is extracted, which is used to compute the similarity between two faces. When the similarity between source model and target model is calculated, we should know how source faces are matched with target faces. Here, a bipartite graph is adopted to solve the optimal match scheme between source faces and target faces.

A bipartite graph is a special graph. $\mathrm{G}=(\mathrm{V}, \mathrm{E})$ is an undirected graph. $\mathrm{V}$ is the set of vertexes and $E$ is the set of edges. $V$ is divided into two disjoint subsets $V_{1}$ and $V_{2}$. Every edge in $\mathrm{E}$ is associated with two vertexes. One vertex belongs to subset $\mathrm{V}_{1}$ and the other belongs to subset $V_{2}$. A matching in a graph is a subset of its edges, and any two edges in matching are not dependent on the same vertex. In a weighted bipartite graph, each edge has an associated value. A maximum weighted bipartite matching is defined as a matching where the sum of edges' values in the matching has a maximum value, which can be solved by Kuhn-Munkras (KM) algorithm.

The method of matching source faces and target faces based on bipartite graph is shown as follows:

(1)Number faces in source model and its number of faces is $\mathrm{m}$. Number faces in target model and its number of faces is $n$.

(2)If $m$ is larger than or equal to $n, m$ is set to $t$. Otherwise, $n$ is set to $t$.

(3)A 2 dimensional matrix $S$ is constructed. $S$ is $n$ order matrix. Its row corresponds to target face and its column corresponds to source face. Use equation (1) to compute the similarity $s_{i j}$ between source face $i$ and target face $j$. The similarity $s_{i j}$ is set to $S[i, j]$.

(4)In order to solve the matching problem conveniently, elements in matrix $S$ are amplified. $S[i, j]=S[i, j] * 1000$.

(5)Use KM algorithm to deal with similarity matrix $\mathrm{S}$ and get the optimal matching scheme between source faces and target faces.

(6) Output source faces and their matched target faces.

For source model $\mathrm{M}_{\mathrm{S}}$ and target model $\mathrm{M}_{\mathrm{T}}$ shown in Figure 3, the proposed method is used to solve source faces and their matched target faces.

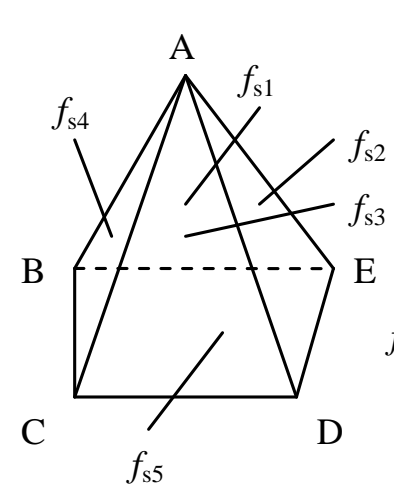

Source model

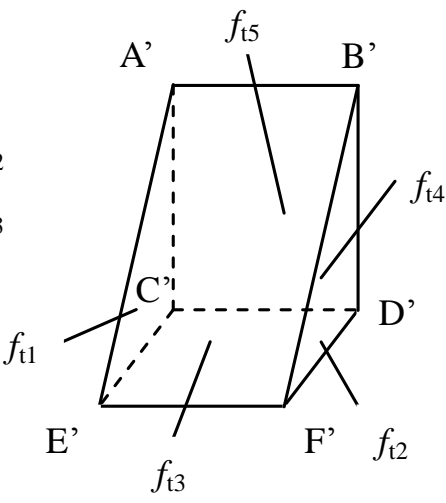

Target model

\section{Figure 3. Source Model $M_{S}$ and Target Model $M_{T}$}

The similarity matrix $S$ is shown in Figure 4. The rows correspond to target faces including $f_{\mathrm{t} 1}, f_{\mathrm{t} 2}, f_{\mathrm{t} 3}, f_{\mathrm{t} 4}$ and $f_{\mathrm{t} 5}$ in target model. The columns correspond to source faces including $f_{\mathrm{s} 1}, f_{\mathrm{s} 2}, f_{\mathrm{s} 3}, f_{\mathrm{s} 4}$ and $f_{\mathrm{s} 5}$ in source model. 


$S=\begin{array}{cccccc} & f_{s 1} & f_{s 2} & f_{s 3} & f_{s 4} & f_{s 5} \\ f_{t 1} & 1000 & 1000 & 1000 & \underline{1000} & 750 \\ f_{t 2} & 1000 & 1000 & \underline{1000} & 1000 & 750 \\ f_{t 3} & 750 & 750 & 750 & 750 & \underline{1000} \\ f_{t 4} & 750 & \underline{750} & 750 & 750 & 1000 \\ f_{t 5} & \underline{750} & 750 & 750 & 750 & 1000\end{array}$

\section{Figure 4. The Similarity Matrix between Source Faces and Target Faces}

KM algorithm is used to deal with similarity matrix $S$ and get the optimal match scheme between source faces and target faces. Target face $f_{\mathrm{t} 1}$ corresponds to source face $f_{\mathrm{s} 4}$ and the similarity is 1000 . Target face $f_{\mathrm{t} 2}$ corresponds to source face $f_{\mathrm{s} 3}$ and the similarity is 1000. Target face $f_{\mathrm{t} 3}$ corresponds to source face $f_{\mathrm{s} 5}$ and the similarity is 1000 . Target face $f_{\mathrm{t} 4}$ corresponds to source face $f_{\mathrm{s} 2}$ and the similarity is 750 . Target face $f_{\mathrm{t} 5}$ corresponds to source face $f_{\mathrm{s} 1}$ and the similarity is 750 . The computation of the similarity between source model $\mathrm{M}_{\mathrm{S}}$ and target model $\mathrm{M}_{\mathrm{T}}$ is shown in Equation (2).

$$
\begin{aligned}
\operatorname{Sim}_{\bmod e l}\left(M_{S}, M_{T}\right) & =1000+1000+1000+750+750 \\
& =4500
\end{aligned}
$$

\section{Experiment}

In order to illustrate the feasibility and validity of the proposed method, four different models are selected in experiments. Target model is shown in Figure 5. Source models are shown in Figure 6, including source model 1, source model 2, source model 3 and source model 4.

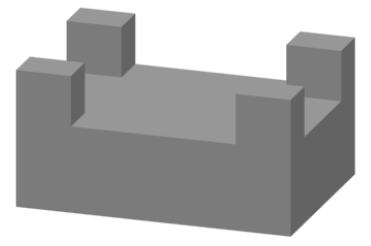

Figure 5. Target Model

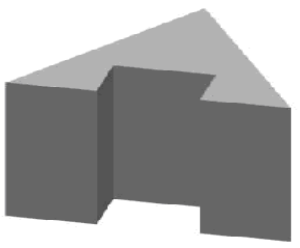

Source model 1

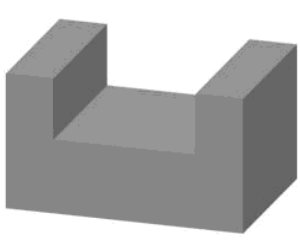

Source model 2

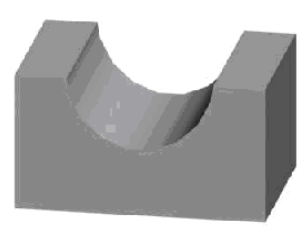

Source model 3

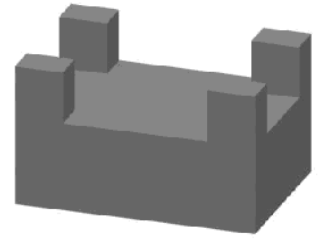

Source model 4

Figure 6. Source Models

Number of vertexes, number of faces and number of edges in source models are shown in Table 2. At the same time, the similarity between source model and target model is also described in Table 2.

\section{Table 2. Similarity between Source Model and Target Model}

$\begin{array}{lllll}\text { Model number } & \text { Number of vertexes } & \text { Number of faces } & \text { Number of edges } & \text { Model similarity }\end{array}$




\begin{tabular}{ccccc}
\hline 1 & 14 & 9 & 21 & 8750 \\
2 & 16 & 10 & 24 & 10000 \\
3 & 12 & 8 & 28 & 8000 \\
4 & 32 & 18 & 48 & 18000 \\
\hline
\end{tabular}

In model 1 , there are 14 vertexes, 9 faces and 21 edges. Its similarity is 8750 . For model 2 , the number of vertexes is 16 , the number of faces is 10 and the number of edges is 24 . Its similarity is 10000 . In model 3 , there are 12 vertexes, 8 faces and 28 edges. Its similarity is 8000 . For model 4 , the number of vertexes is 32 , the number of faces is 18 and the number of edges is 48 . Its similarity is 18000 . The experimental results show that source model 4 is the same with target model in shape and their similarity is highest. Source model 2 is very similar with target model in shape and their similarity is higher. Source model 1 and source model 3 are not similar with target model in shape. Their similarities are low. So, the proposed method can evaluate the shape similarity between source model and target model.

\section{Conclusions}

In this paper, attribute adjacent graph is applied to describe CAD models. The equation of computing the similarity between two faces is given. To compute the similarity of models is changed into calculating similarities of faces. A bipartite graph is used to describe the matching problem between source faces and target faces. At the same time, a new method to solve the optimal matching scheme between source faces and target faces is given. Experimental results show that this method can evaluate the shape similarity between source model and target model.

\section{Acknowledgements}

This work is supported by Science and Technology Research Funds of Education Department in Heilongjiang Province under Grant Nos. 12541125.

\section{References}

[1]. R. Huang, S. S. Zhang, X. L. Bai, K. X. Zhang and X. M. Zhang, "Manufacturing semantics based 3D CAD model retrieval method", Computer Integrated Manufacturing Systems, vol. 19, (2013), pp. 626629.

[2]. K. X. Zhang, S. S. Zhang and L. Li, "A method of 3D CAD model retrieval based on ant colony algorithm", Journal of Computer-Aided Design \& Computer Graphics, vol. 23, (2011), pp. 633-639.

[3]. S. Q. Tao, Z. D. Huang and T. G. Zheng, "3D CAD model retrieval based on attributed adjacency graph matching", Computer Integrated Manufacturing Systems, vol. 17, (2011), pp. 680-687.

[4]. S. Q. Tao, S. T. Wang, T. G. Zheng and Z. D. Huang, "CAD model retrieval based on inexact graph matching", Journal of Computer-Aided Design \& Computer Graphics, vol. 22, (2010), pp. 545-552.

[5]. W. Sun, T. Q. Ma, T. Li and T. M. Su, "Partial retrieval of CAD models based on topology approximation”, Journal of Computer-Aided Design \& Computer Graphics, vol. 21, (2009), pp. 18051813.

[6]. L. J. Ma, Z. D. Huang and Q. S. Wu, "Retrieval of CAD models based on shape-location codes of faces", Journal of Computer-Aided Design \& Computer Graphics, vol. 20, (2008), pp. 19-25.

[7]. J. Bai, S. M. Gao and W. H. Tang, "Design reuse oriented partial retrieval of CAD models", CAD Computer Aided Design, vol. 42, (2010), pp. 1069-1084.

[8]. W. Liu and Y. J. He, "Representation and retrieval of 3D CAD models in parts library", International Journal of Advanced Manufacturing Technology, vol. 36, (2008), pp. 950-958.

[9]. F. Bosche and C. T. Haas, "Automated retrieval of 3D CAD model objects in construction range images", Automation in Construction, vol. 17, (2008), pp. 499-512.

[10].M. M. Marefat and C. D. Pitta, "Similarity-based retrieval of CAD solid models for automated reuse of machining process plans", Proceedings of the 3rd IEEE International Conference on Automation Science and Engineering, Scottsdale, (2007), pp. 312-317.

[11].L. Li, H. Z. Wang and T. J. Chin, "Retrieving 3D CAD models using 2D images with optimized weights", Proceedings of 2010 3rd International Congress on Image and Signal Processing, Yantai, (2010), pp. 1586-1589. 
[12].B. K. Hu, Y. S. Liu and S. M. Gao, "Parallel global optimal approach of feedback for 3D CAD model retrieval", Proceedings of the 2007 10th IEEE International Conference on Computer Aided Design and Computer Graphics, Beijing, (2007), pp. 132-137.

\section{Authors}

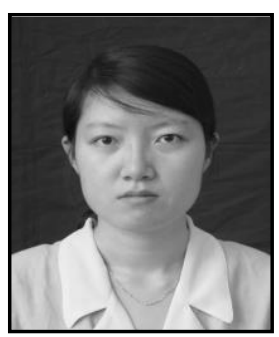

Xue-Yao Gao is Ph.D. She is also an associate professor in Harbin University of Science and Technology. Her research interests are CAD and model retrieval. She has authored and coauthored more than 20 journal and conference papers in these areas.

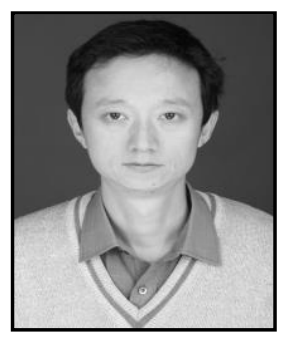

Chun-Xiang Zhang is $\mathrm{Ph} . \mathrm{D}$. He is also a professor in Harbin University of Science and Technology. His research interests are $\mathrm{CAD}$ and machine learning. He has authored and coauthored more than 50 journal and conference papers in these areas. 
International Journal of Signal Processing, Image Processing and Pattern Recognition Vol. 8, No. 2 (2015) 